\title{
PROPOSAL FOR A RAILWAY LAYER MODEL
}

\author{
MARTIN SCHEIDT \\ TU Braunschweig, Institute of Railway Systems Engineering and Traffic Safety, Germany
}

\begin{abstract}
In railway systems, timetables and infrastructure are key components for satisfying transport needs. In order to find a feasible configuration that satisfies the complex set of requirements, numerous timetables and track topologies have to be evaluated. Therefore, there is an immense need for automatic timetable and track topology design. This paper discusses the concept of creating abstraction layers based on a data model to support and simplify these designs. It uses different state-of-the-art models and combines them so that all aspects of railway operation are included. Using this method, a layer model has been developed that was inspired by the OSI Reference Model and takes current developments such as the UIC RailTopoModel into consideration. Possible applications of such a layer model are the automatic generation of timetables and track topologies, as well as entrance data for synchronous railway simulations for capacity evaluation, and a universal representation of a railway network.

Keywords: railway model, layer model, infrastructure model, timetable model, transport model, transit model, interlocking model, speed profile.
\end{abstract}

\section{INTRODUCTION}

In order to conduct railway operations research and evaluate railway networks, a considerable amount of data, from infrastructure to timetables, must be analysed. Managing and organising all the data is key to the success of such evaluations. This article presents a universal model to process this data. This model includes various aspects of a railway system, from infrastructure to the services running on it, in order to obtain a complete representation of the data involved. To do this, several existing models have been examined and incorporated into a combined model. Many of these models emphasise different aspects of railway systems; therefore, the primary task is to break down a railway system and identify aspects that need to be included in the overall model.

The following chapter will present the motivation for this research and explain why a layered model can assist in solving specific problems. Subsequently, this paper will show how to divide a rail system into layers and highlight specific functions for each component. Using these components, chapter four will present a railway layer model in which each layer will be discussed in more detail and related back to existing models. The paper will then be concluded with a look at further research on the topic.

\section{MOTIVATION}

The infrastructure and the timetable applied to it form the basis for every railway system. Both of these elements have to be developed to fulfil traffic demands. Because they are strongly linked, this leads to a "chicken-and-egg" dilemma, which means that they cannot be designed independently of each other. A tool to calculate infrastructure, and thus track topology, from a timetable and vice versa could help facilitate a solution to the dilemma. The computation of a timetable from a given track topology is well known, but the reverse remains in need of improvement.

First, the logical basis of timetabling will be examined in order to address both directions of this computation. The classical approach to timetabling is running time estimations with a

ORCID: https://orcid.org/0000-0002-9384-8945 


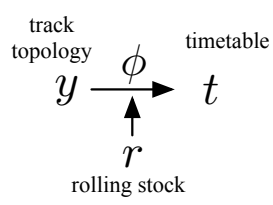

(a)

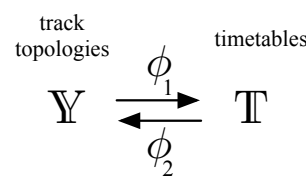

(b)

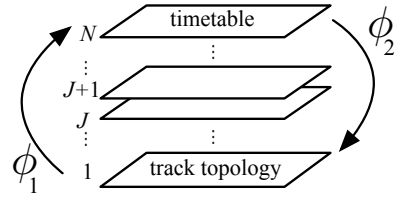

(c)

Figure 1: Transitions. (a) Classical running time estimation; (b) Generic approach; (c) Layers to reduce the solution space.

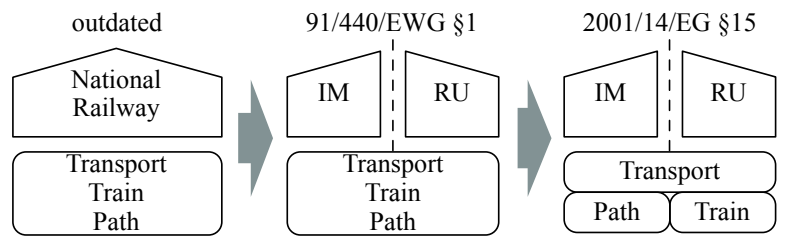

Figure 2: Division of a national railway between infrastructure manager (IM) and railway undertaker (RU) requested by European legislation based on [2].

specific rolling stock (see Fig. 1(a)). Many attributes of infrastructure elements (the physical connections from the track topology) and vehicle formations (composed of rolling stock) have to be examined for the computation of the timetable (combined time slots of individual train movements). If a specific rolling stock is reduced to performance parameters (see [1]) and substituted using the concept of paths (time slots for specific performance parameters), a more general approach to timetabling could gain the ability to conclude track topology from a proposed timetable. The concept of paths is even enforced by European legislation through the division of infrastructure manager and railway undertaker (see Fig. 2). This reduction to a path emphasises the transformation $(\phi)$ and can be extended to the whole set of track topologies and timetables (see Fig. 1(b)). There are two transformation directions: $\phi_{1}$ from set track topologies $(\mathbb{Y})$ to set timetables $(\mathbb{T})$ and $\phi_{2}$ from set timetables $(\mathbb{T})$ to set track topologies $(\mathbb{Y})$.

\section{$2.1 \phi_{1}$ : From track topology to timetable}

The transition from $\mathbb{Y}$ to $\mathbb{T}$ is the timetabling process. One specific track topology can have more than one corresponding timetable, and one specific timetable could fit more than one track topology. This indicates that the mathematical solutions to the transition between the sets $\mathbb{Y}$ and $\mathbb{T}$ will be vast. However, many solutions will not be plausible from a railway perspective. A specific transition from one track topology to one or more timetables is stateof-the-art and already implemented in many railway-related software tools.

\section{$2.2 \phi_{2}$ : From timetable to track topology}

The formal transition from $\mathbb{T}$ to $\mathbb{Y}$ is neither commonly known nor used. This transition could be useful to determine infrastructure insufficiencies for the planning of integrated clock-face timetables. Just as with the transition $\phi_{1}$, the transition $\phi_{2}$ will result in a vast amount of 
solutions, some of which will be suitable for railway application. This indicates that the primary task will be to reduce the solution space.

\subsection{Reduction of solution space}

The solution space of the transition $\phi_{1}$ is mainly determined by the characteristics of a path and is guided by operational constraints. However, the solution space of the transition $\phi_{2}$ is not specifically determined and needs to be restricted. A reverse calculation of the riding dynamics could be applied for this restriction, but due to its complex mathematical nature, it might not be practicable.

A different idea is to further restrict the transition $\phi_{2}$ by introducing abstraction layers as a guide for reducing complexity. Layers of abstraction were determined by following the transition $\phi_{1}$ and forming a layer model (see Fig. 1(c)). A layer model comes with certain advantages and disadvantages. The main weakness of layers is the emergence of additional and perhaps unnecessary forms of abstraction and interdependencies. However, it is possible to simplify an aspect of a single layer. This level-based simplicity helps to optimise and transform aspects of a layer without losing the overall context. The linking between the layers also can contribute to recognise interdependencies. Furthermore, a layer model is ideal for a large system with mixed low- and high-level functions (see Buschmann et al. [3]).

According to Buschmann et al. [3], decomposition should be approached by structuring the system into an appropriate number of functions. Then, the lowest level of function (layer 1) should be placed at the base of the system. Intermediate steps are termed layer $J$ and build upon each other $(J+1)$ until the top level of functionality is reached with layer $N$. The OSI-Model [4] represents a classic example of level division in this manner.

The UIC RailTopoModel standard [5] briefly mentions a layer model and encourages its enrichment and further development (see Fig. 3(a)). Unfortunately, the UIC RailTopoModel only targets the track topology of a railway network but not the timetable aspect and therefore cannot cover the other aspects of railway systems. To model the railway system with a more holistic view, a comprehensive model in which the complete system is decomposed into different components and analysed needs to be developed. Current state-of-the-art models focus on individual aspects. Therefore a combination of those models together with a comparison of the decomposed components leads to a comprehensive model.

\section{DECOMPOSING THE RAILWAY SYSTEM}

Bosse [6] describes the railway system with four major components: the tracks and terminals, the rolling stock, operational rules and procedures, and signalling (see Fig. 3(b)). All components have functions. Together, this results in six functional tiers: transport, network, access, safety regulation, traffic regulation, and interlocking. The rolling stock accommodates the transport on the rail network via a path. Tracks and terminals serve the path on a physical network and the access to the network. Operational rules and procedures implement safety regulation and higher traffic regulation. Signalling maintains the complex safety with interlocking. Thus the complete system is formed by the components and their functions.

\subsection{Ordering the components}

It is necessary to identify the relationship and the dependencies between each layer to order the tiers, specifically, which services are provided by layer $J$ and then used by layer $J+1$.

Following the UIC RailTopoModel, we must first establish the topology as the base of the model. The topology is provided by the network tier and represents physical connections and 


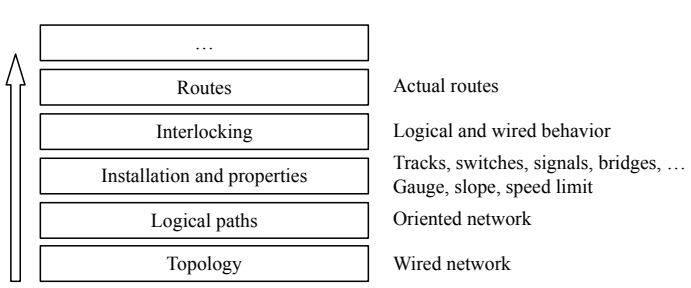

(a) Proposed layers by UIC RailTopoModel [5].

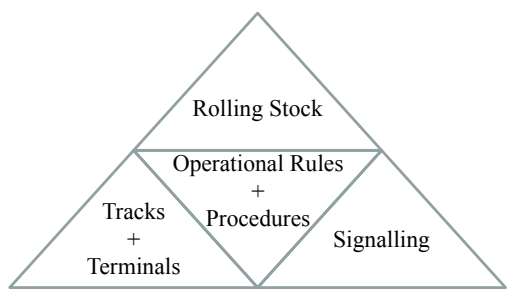

(b) Railway system according to [6].

Figure 3: UIC RailTopoModel and components of railway systems.

Table 1: Proposed layers for the railway model.

\begin{tabular}{clll}
\hline Layer & Name & Content & Task \\
\hline 0 & Base & Macroscopic network & Regulation, Network \\
1 & Physical & Basic properties, Connections & Topology \\
2 & Speed Profile & Speed restriction, Resistance & Running time estimation \\
3 & Interlocking & Blocks, Routes, Train control & Safety \\
4 & Resource & Queue network, Dispatching rules & Utilisation \\
5 & Transit & Path, Halts, Interval & Timetable \\
6 & Transport & Rail services & Shipment, Travel \\
\hline
\end{tabular}

attributes, such as speed, radius, gradient, coordinates and attached objects. These attributes are mostly independent of a specific train or path except for the speed profile (see Section 4.3). Two layers are proposed for the network tier to remove the path-related attributes from the topology and combine information for running time estimations. These two layers are: a Physical Layer with fundamental properties and connections for the topology and a Speed Profile Layer with speed restrictions and resistances for running time estimations.

The information about the permitted speed from the Speed Profile Layer needs to be transmitted to a running train to ensure speed restrictions. The interlocking tier provides such functionality, including the authority of general movement which contains blocks, routes, and train control. Therefore, an Interlocking Layer is placed on top.

Next, the traffic regulation tier needs to be established, since the Interlocking Layer introduces blocks and, along with them, possible occupations. The addition of a Resource Layer can utilise a queue network and ensure deadlock prevention with the functionality of dispatching.

On top of the Resource Layer, paths with blocking time theory can be constructed. Paths are used for the transits between terminals and subsequently to construct timetables. The usefulness of the paths depends on access to terminals, their intervals and stopping patterns. Hence, a Transit Layer is proposed to accommodate paths.

Rail services can then be planned and operated with the provided paths from the Transit Layer. These services are used to fulfil the transport needs of passengers or goods. Additionally, a Transport Layer is introduced to enable the optimisation of transport chains.

At this point, the only missing component is the safety regulations tier. Therefore, an encompassing Base Layer is placed below all previous layers to accommodate regulation rules and other meta information, such as the macroscopic network, the operator and the ownership. 


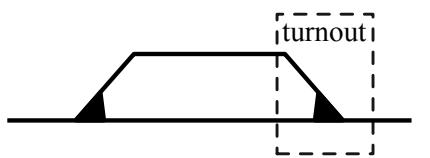

(a)

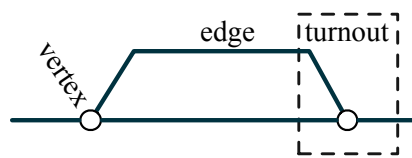

(b)

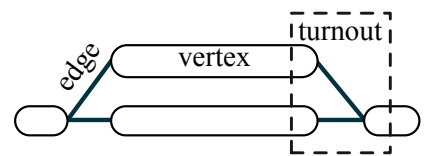

(c)

Figure 4: Graph modelling. (a) Exemplary track layout; (b) Turnout as a vertex; (c) Turnout as edges.

Table 1 shows the summary of all layers with their order number, content and tasks. This model represents a relaxed layer system, which means there is no restrictive relationship between layers. Each layer may use the services of all of the layers below it, not only the services of the layer immediately below [3]. For example, the Speed Profile Layer provides speed information not only for the Interlocking Layer, but also for the Transit Layer in order to calculate paths. Each layer is discussed in further detail in Section 4 and a full example can be found in Fig. 11.

\subsection{Joining nodes}

The joining nodes define the relationship between the layers. Most of the representations share a branching at a turnout. Therefore, the primary joining nodes will be turnouts in the layer model.

Railway models built upon graphs (ordered pair $G=(\mathbb{V}, \mathbb{E})$ containing a set $\mathbb{V}$ of vertices with a set $\mathbb{E}$ of edges) naturally support the structure of a railway network. Therefore, graphs are used where applicable. There are two general possibilities for modelling a turnout: as a vertex (see Fig. 4(b)) or as two edges (see Fig. 4(c)). Both types of modelling are used by the layer model to provide different aspects of the model. Furthermore, a simple undirected graph will not be sufficient to encompass all characteristics inside a layer and thus will be extended and modified when needed.

\subsection{Base layer}

\section{RAILWAY LAYER MODEL}

As mentioned in Section 3.1 and Table 1, the Base Layer contains meta information about safety regulations and the ownership of the infrastructure. The layer's task is to gather information and provide this information in relation to the macroscopic network. This information can also contain names and register information of operational points and their connections.

\subsection{Physical layer}

The Physical Layer embodies the actual physical infrastructure of the railway. This layer has to cope with the specific constraints of the track topology. The constraints are:

- direction dependence,

- impossibility of turns, and

- mutual exclusion. 


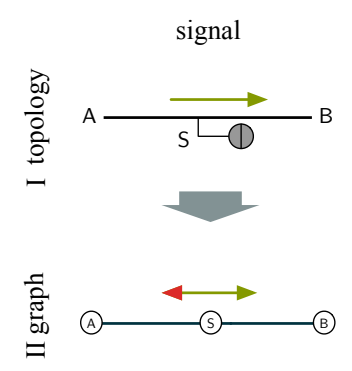

(a)

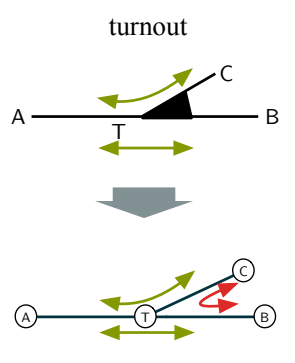

(b)

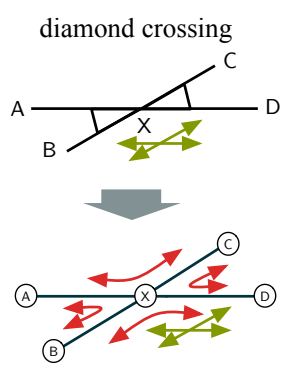

(c)

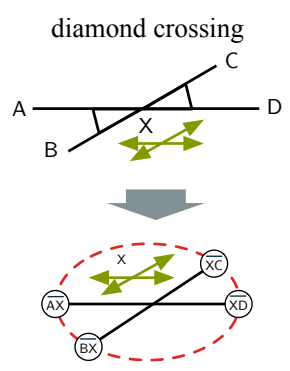

(d)

Figure 5: Constraints of the track topology. (a) Direction dependence; (b) and (c) Impossible turn; (d) Mutual exclusion.

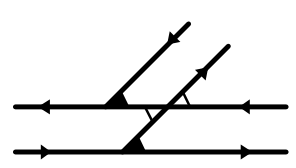

(a)

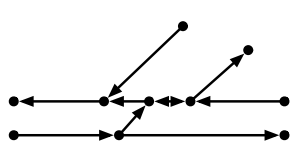

(b)

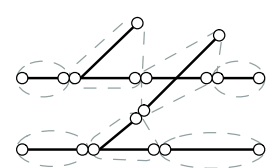

(c)

Figure 6: An exemplary track layout and its representation in different models. (a) Exemplary track topology; (b) Directed graph [7]; (c) Double vertex graph [8].

These constraints result from three different common elements: a signal, a turnout and a diamond crossing. The signal is only readable in one direction and has no significance for the other. At a turnout, a course change is only possible on the pointed side. At a diamond crossing, discounting impossible turns, two trains cannot pass at the same time. Fig. 5 shows a simple graph for every corresponding restriction.

Völz [7] transfers the rail infrastructure to a simple directed graph to depict the connected tracks. However, this modelling includes the problem of the impossible turn, which has to be handled on a higher level of program logic. Montigel [8], on the other hand, uses a double vertex graph to overcome this problem. Fig. 6 shows an exemplary track layout and illustrations of all previously-mentioned models. Montigel also introduces formal track topology, including a solution for the mutual exclusion problem at diamond crossings. Therefore, the double vertex graph is well-suited as a model for the Physical Layer since it can handle all railway infrastructure constraints.

The Physical Layer will include properties such as track gauge, electrification and loading gauge, which can be encoded either on the vertex or the edge. The properties of curvature and gradient could be stored as well, but carry a special meaning for running time estimations. They can either provide the data as a service for the next layer or can be included in it.

\subsection{Speed profile layer}

The task for the Speed Profile Layer is to enable running time estimations, which requires two parts: track resistance and speed restrictions. Track resistance results from the gradient and the curvature of the track itself, which can either be stored in a graph or retrieved by a service, depending on the implementation in the Physical Layer. Speed restriction, on the other hand, 


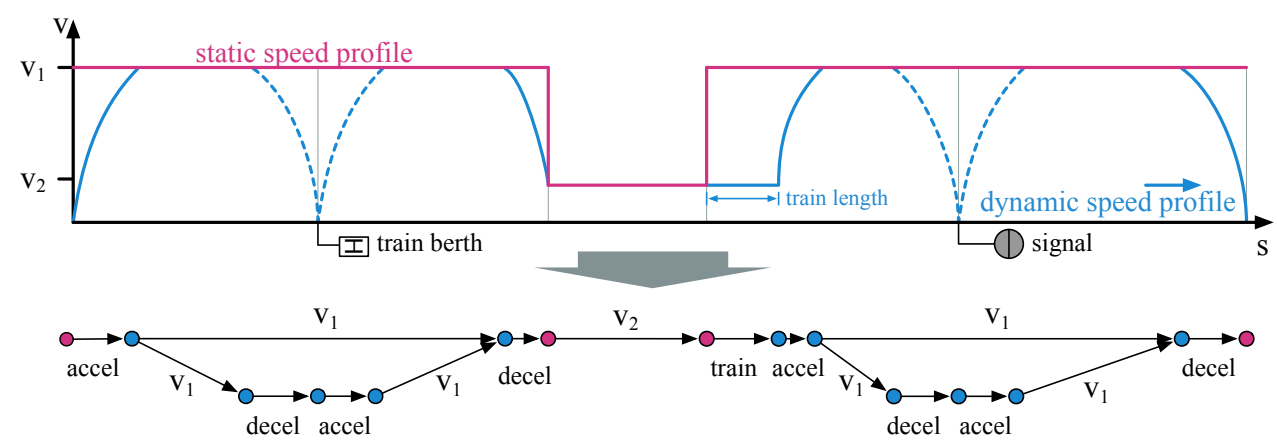

Figure 7: Static speed profile and dynamic speed profile. Compare [9] and [10].

follows two different limitations (see Fig. 7). The first limitation is a consequence of the physical constraints that result mainly from curvature; the second limitation results from the movement of a train. This produces two profiles: a static speed profile and a dynamic speed profile.

The static speed profile addresses the maximum permitted speed at any location, including a scaling problem between micro-, meso- and macroscopic models, as described by Radtke [9]. The dynamic speed profile considers the possible acceleration or deceleration curve of the movement of the train. To specify the curve, generic characteristics for trains can be used. However, these characteristics should match the characteristics of the path constructed from it.

A vehicle does not necessarily need to fully use a maximum speed in the profile and a train protection system commonly oversees the permitted speed. The information about the movement can be encoded either on the edge or on the vertex.

\subsection{Interlocking layer}

According to Theeg and Vlasenko [10], the purposes of interlocking systems are:

- providing speed limits for train protection,

- granting routes and movement authorities, and

- realising track clear detection.

The Speed Profile Layer already provides speed limits, which leaves routes, movement authorities and track clear detection to be addressed by the Interlocking Layer. Since there is a diverse range of technical implementations, no widely accepted models are available. However, there are specific conditions that have to be met with every application.

A route has to safeguard two aspects to secure a running path for a train: the blocking function and the interlocking of moveable track elements. Both aspects can be transformed into a graph as shown in Figs 8(a) and 8(b). Commonly, both route aspects are combined in a single route object with different parts: a running path, an overlap, a flank area, an end section and a start section [10]. Fig. 8(c) illustrates these parts in their topological order and their transformation into a graph. With this graph, the Interlocking Layer can accommodate routes and blocks.

The track clear detection is represented by the clearing points from routes and blocks. These clearing points divide a track into separated track sections that service the next layer. 


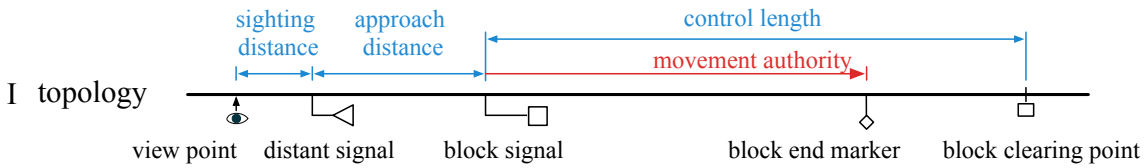

II graph

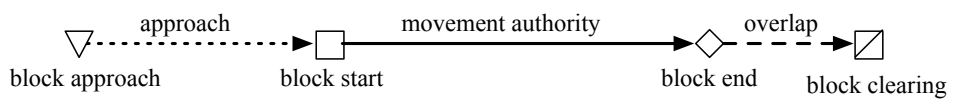

(a) The aspect mutual exclusion as blocking function.

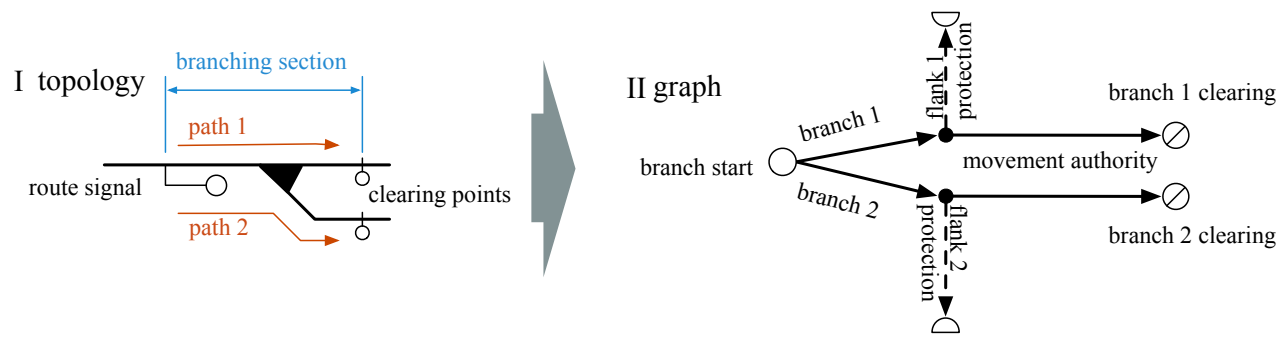

(b) The aspect branching as interlocking function.

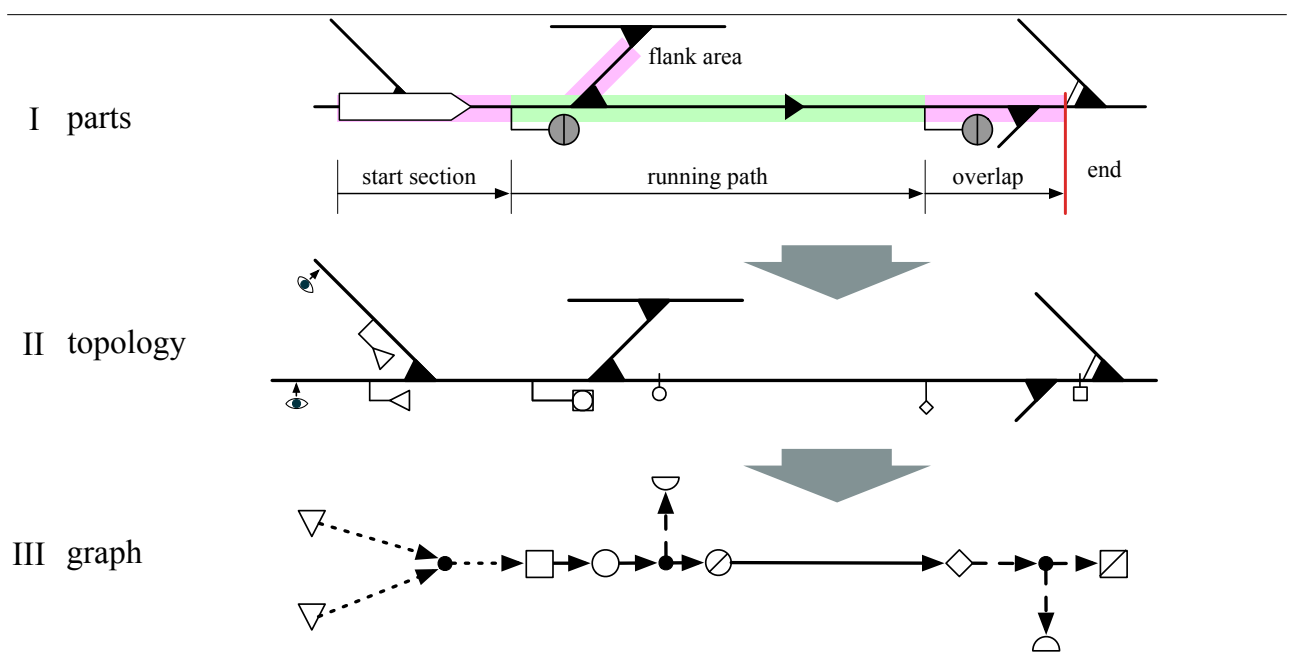

(c) Route transformation with combined aspects (I is based on [10]).

Figure 8: Route aspects and their transformations. 


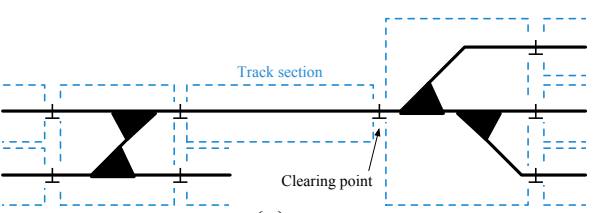

(a)
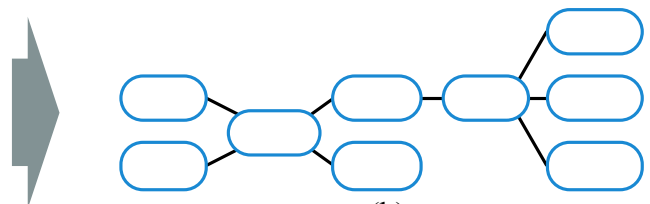

(b)

Figure 9: (a) Clearing points are dividing tracks into sections; (b) Connexity graph [11].

\subsection{Resource layer}

Track sections divided by clearing points can be transformed into a connexity graph (see Fig. 9). Gély et al. [11] use the aforementioned graph as a model of resources (such as route sections, platforms or ways). Also, Montigel's [8] model includes these resources as equivalence classes (see Section 4.2). This concept of resources can serve the specific problem of mutual exclusion and form the Resource Layer.

The resulting queue network of connections can be occupied by a moving train according to blocking time theory [12]. The primary task of the Resource Layer is to utilise the queue network. Additionally, the functionality of dispatching is required to ensure deadlock prevention.

\subsection{Transit layer}

Paths are vital for organising traffic on top of the queue network. Basic blocking time theory can be used for the construction of paths, but it does not solve a further problem: the variability of train movements. Medeossi et al. [1] extend basic blocking time with performance parameters to include these variations. Hertel and Steckel [13] illustrate that these parameters also vary significantly between passenger and freight trains. Therefore, different performance parameters are necessary in order to construct paths with blocking times for different types of trains. The different paths can then be used by either passenger or freight trains.

Furthermore, Pöhle [14] shows that different interval and stopping patterns need to be considered for the operating varieties. Both operating varieties for passenger and freight trains can be divided into fixed patterns, partly fixed patterns, and no existing patterns. Although this leads to nine types of path classification (see Fig. 10(a)), two major concepts can be used to accommodate them all.

Caimi et al. [15] introduces a concept for a timetable with partial periodicity, which consists of all paths (periodic or non-periodic), including the terminals for the stopping pattern. However, this concept fails to address the paths in which it is only known during operation if a train must temporarily stop at a train berth or not. In this case, the train order has to be changed by overtaking. Pöhle [14] describes a solution for overtaking with the concept of snippets. Snippets are atomised templates for understanding the various situations during different train runs, which consist of fragments for acceleration, deceleration and normal running. Together, they form a train run for a specific circumstance by selecting the suitable snippets.

All paths and path snippets, together with terminals, form the Transit Layer. It handles the nodes in a rail network where either passengers or goods can access a train via a terminal. 

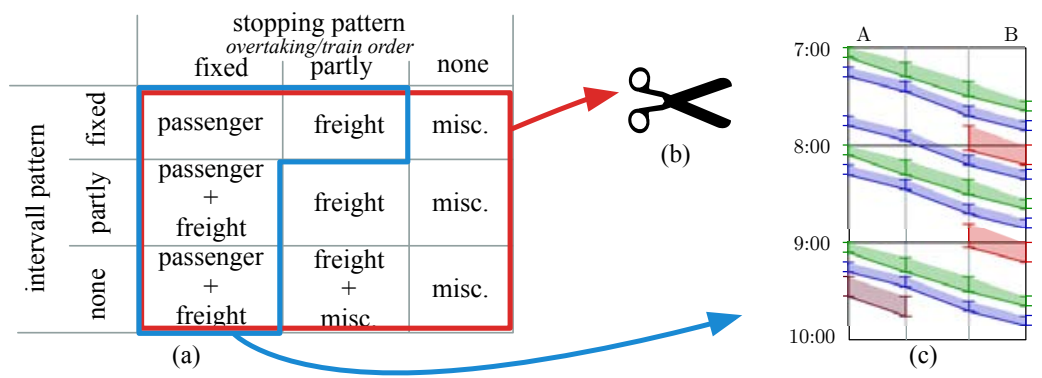

Figure 10: (a) Classifications of paths for central Europe; (b) Snippets [14]; (c) Partial periodic timetable [15].

The layer could also include rules and possible substitutions for specific access points in case operations are disturbed or altered.

\subsection{Transport layer}

The last layer gathers the rail services that are running on a network and provides a timetable for these services. This layer needs to address not only the train runs but also the connections for passengers, staff, vehicles and, if applicable, restraints on the transfer of freight. The model proposed by Caimi et al. [15], which has already been mentioned in Section 4.6, could be used to model train runs, passenger connections and dependencies between trains. This model is well-suited for the Transport Layer, except in the regards of periodicity and the dependencies between trains. The dependencies between trains/paths have already been addressed in the Transit Layer. Periodicity is problematic for non-periodic services, but could still be used for a partially-periodic case as Caimi et al. suggest.

The Transport Layer can be used primarily for the optimisation of services (e.g. PESP and other algorithms). The solution could then be applied to the slots of the Transit Layer.

\section{CONCLUSION AND FURTHER RESEARCH}

In summary, it is possible to create a layer model for the railway system. Currently, the layer model consists of a collection of different models that have been joined together, but it shows the linking and the interdependencies of these models. The closer examination of these models has shown that they emphasise various aspects of the railway system (see Fig. 11). For each layer, a specific aspect and model were collated, and for the interlocking layer, a prototype of a graph based interlocking model was shown. In addition to this research, a proof of concept along with real-world examples are needed to help with the further development and evolution of the layered structure.

A strict layer model seemed not to be suitable for railway systems since there are some properties which are bypassed by intermediate layers (see Section 3.1). Because this research represents a relaxed layer system an ideal order of the layers, therefore, remains to be determined.

The Railway Layer Model tries to include the purpose of a railway service into the view of the infrastructure, in contrast to the UIC RailTopoModel, which focuses on track topology and data structure. Furthermore, more research is required to determine if the architecture into tiers could make railway operations more transparent for track topology planning purposes (transition $\phi_{2}$; see Section 2). 
(a)

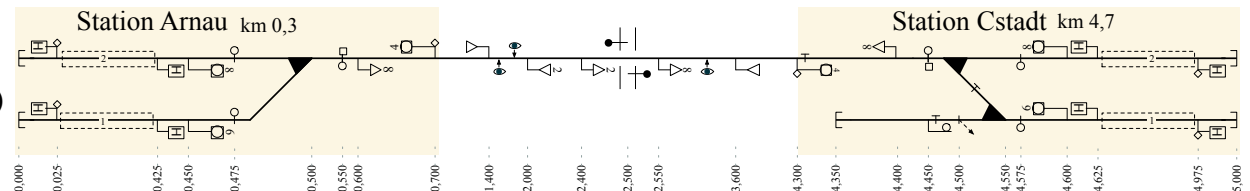

(b)

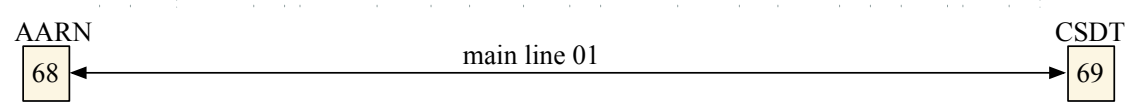

(c)

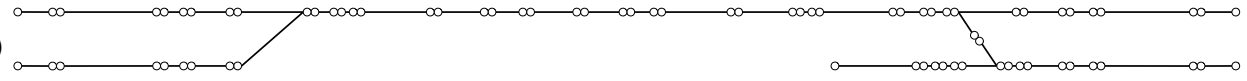

(d)
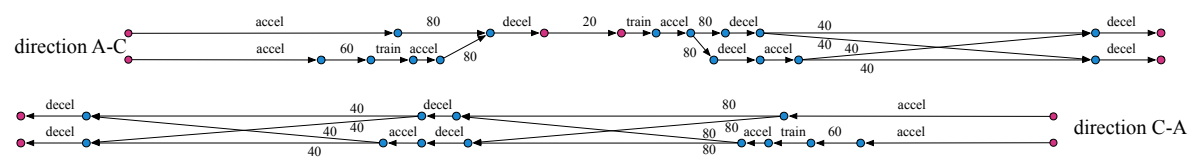

(e)

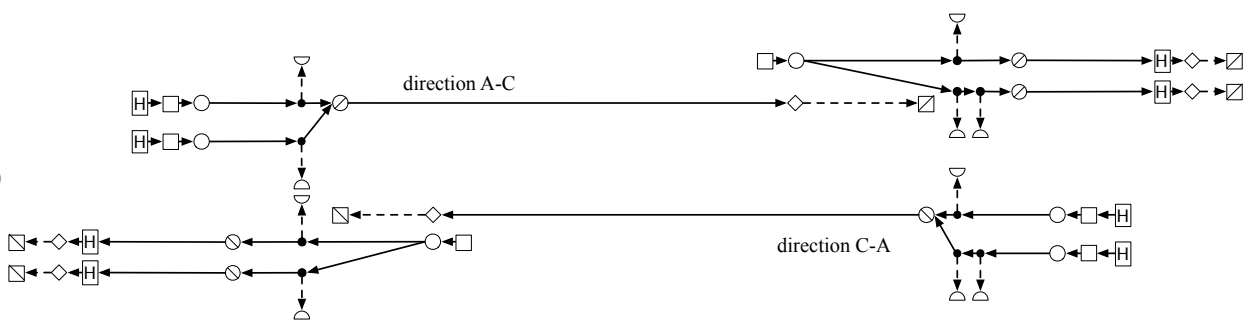

(f)
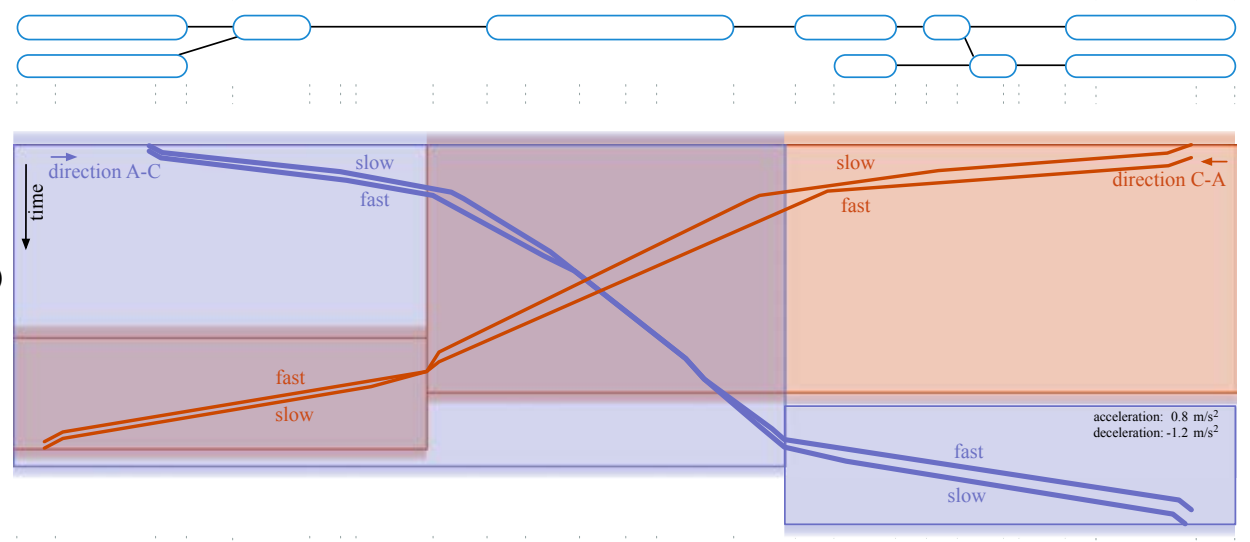

(h)

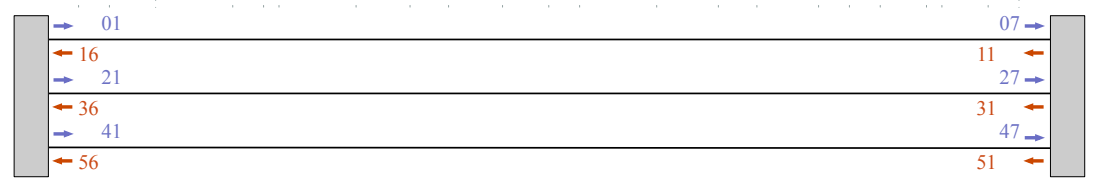

Figure 11: Layer model example. (a) Topology example based on [16]; (b) Base Layer; (c) Physical Layer; (d) Speed Profile Layer; (e) Interlocking Layer; (f) Resource Layer; (g) Transit Layer; (h) Transport Layer (visualisation based on [17]). 


\section{ACKNOWLEDGEMENTS}

We acknowledge support by the German Research Foundation and the Open Access Publication Funds of the Technische Universität Braunschweig.

\section{REFERENCES}

[1] Medeossi, G., Longo, G. \& de Fabris, S., A method for using stochastic blocking times to improve timetable planning. Journal of Rail Transport Planning \& Management, 1(1), pp. 1-13, 2011.

[2] Hantak, H., TAF TSI IM/RU Cluster - TAF TSI Working Group 10 - TAF/TSI Object Identifiers - Handbook. Technical report, RailNetEurope, 2011.

[3] Buschmann, F., Meunier, R., Rohnert, H., Sommerlad, P. \& Stal, M., Layers. PatternOriented Software Architecture, A System of Patterns, John Wiley \& Sons Incorporated: Chichester, UK, pp. 31-51, 1996.

[4] Zimmermann, H., OSI Reference Model-The ISO Model of Architecture for Open Systems Interconnection. IEEE Transactions on Communications, 28(4), pp. 425-432, 1980.

[5] International Union of Railways (UIC), UIC International Railway Standard IRS 30100 RailTopoModel, 2016.

[6] Bosse, G., Grundlagen für ein generisches Referenzsystem für die Betriebsverfahren spurgeführter Verkehrssysteme. PhD thesis, TU Braunschweig, 2010.

[7] Völz, W.D., Ermittlung der Leistungsfähigkeit von Knotenpunkten spurgeführter Verkehrssysteme mittels Graphentheorie. $\mathrm{PhD}$ thesis, Lehrstuhl und Institut für Verkehrswesen, Eisenbahnbau und -betrieb; Technische Universität Hannover, Hannover, 1976.

[8] Montigel, M., Formal Representation of Track Topologies by Double Vertex Graphs. Computers in Railways III, eds. T.K.S. Murthy, J. Allan, R.J. Hill, G. Sciutto \& S. Sone, Washington, DC, pp. 359-370, 1992.

[9] Radtke, A., Infrastructure modelling. Railway Timetabling \& Operations, DVV Media Group GmbH, Eurailpress: Hamburg, pp. 47-63, 2014.

[10] Theeg, G. \& Vlasenko, S., Railway signalling interlocking, DVV Media Eurailpress, Hamburg, 2009.

[11] Gély, L., Dessagne, G. \& Pesneau, P., A multi scalable model based on a connexity graph representation. Computers in Railways XII, Beijing, China, volume 1, pp. 193204, 2010.

[12] Happel, O., Sperrzeiten als Grundlage für die Fahrplankonstruktion. ETR Eisenbahntechnische Rundschau, 8(2), pp. 79-90, 1959.

[13] Hertel, G. \& Steckel, J., Eine neue Philosophie der Fahrzeitberechnung für Zugfahrten. Wissenschaftliche Zeitschrift Hochschule für Verkehrswesen Friedrich List, pp. 104111, 1992.

[14] Pöhle, D., Strategische Planung und Optimierung der Kapazität in Eisenbahnnetzen unter Nutzung von automatischer Taktfahrplanung. PhD thesis, Hamburg, 2016.

[15] Caimi, G., Laumanns, M., Schuepbach, K., Woerner, S. \& Fuchsberger, M., The periodic service intention as a conceptual framework for generating timetables with partial periodicity. Transportation Planning and Technology, 34(4), pp. 323-339, 2011.

[16] railML.org e.V., Simple example data set (v0.7) of railway infrastructure modelled in railml 2.3 and in railml 3.1beta. www.railml.org/en/user/exampledata.html. Accessed on: 16 Feb. 2018.

[17] SMA und Partner AG, Netgraph timetable Switzerland. www.smapartner.com/en/about-sma/downloads, Accessed on: 16 Feb. 2018. 